\title{
Review Article \\ Genes Associated with SLE Are Targets of Recent Positive Selection
}

\author{
Paula S. Ramos, ${ }^{1}$ Stephanie R. Shaftman, ${ }^{2}$ Ralph C. Ward, ${ }^{2}$ and Carl D. Langefeld ${ }^{3}$ \\ ${ }^{1}$ Department of Medicine, Medical University of South Carolina, Charleston, SC 29425, USA \\ ${ }^{2}$ Department of Public Health Sciences, Medical University of South Carolina, Charleston, SC 29425, USA \\ ${ }^{3}$ Department of Public Health Sciences, Wake Forest School of Medicine and Center for Public Health Genomics, \\ Winston-Salem, NC 27157, USA \\ Correspondence should be addressed to Paula S. Ramos; ramosp@musc.edu
}

Received 23 September 2013; Accepted 12 November 2013; Published 23 January 2014

Academic Editor: Juan-Manuel Anaya

Copyright (c) 2014 Paula S. Ramos et al. This is an open access article distributed under the Creative Commons Attribution License, which permits unrestricted use, distribution, and reproduction in any medium, provided the original work is properly cited.

\begin{abstract}
The reasons for the ethnic disparities in the prevalence of systemic lupus erythematosus (SLE) and the relative high frequency of SLE risk alleles in the population are not fully understood. Population genetic factors such as natural selection alter allele frequencies over generations and may help explain the persistence of such common risk variants in the population and the differential risk of SLE. In order to better understand the genetic basis of SLE that might be due to natural selection, a total of 74 genomic regions with compelling evidence for association with SLE were tested for evidence of recent positive selection in the HapMap and HGDP populations, using population differentiation, allele frequency, and haplotype-based tests. Consistent signs of positive selection across different studies and statistical methods were observed at several SLE-associated loci, including PTPN22, TNFSF4, TET3DGUOK, TNIP1, UHRF1BP1, BLK, and ITGAM genes. This study is the first to evaluate and report that several SLE-associated regions show signs of positive natural selection. These results provide corroborating evidence in support of recent positive selection as one mechanism underlying the elevated population frequency of SLE risk loci and supports future research that integrates signals of natural selection to help identify functional SLE risk alleles.
\end{abstract}

\section{Introduction}

Systemic lupus erythematosus (SLE) is an autoimmune disease whose prevalence, incidence, and disease severity are known to vary among ethnic groups. Increased prevalence has been reported among African-Americans, Asians, Hispanics, and Native Americans (reviewed elsewhere $[1,2]$ ). The reasons for the ethnic disparities remain elusive. According to the "hygiene hypothesis" first proposed by Strachan two decades ago [3], the increased disease prevalence of autoimmune and allergic diseases in industrialized countries may be due to modern society's limited pathogen exposure. The Hygiene Hypothesis posits that humans have adapted to infectious exposures that were the norm in the past and that exposure was protective against autoimmune disease. Over many generations environmental pressure may have favored alleles that allow humans to respond to immune system challenges differently but resulted in an increased risk of autoimmune diseases. This could be a mechanism explaining the number of SLE risk alleles that are common in the population.

Human genome variation at the population level is shaped by four evolutionary processes: mutation, migration, random genetic drift, and natural selection. Natural selection is the process by which a trait, in the context of the organism's environment, becomes either more or less common in a population as a function of the effect of the inherited trait on the differential reproductive success. This ability to survive and reproduce and contribute to the gene pool of the next generation is known as fitness. Natural selection drives adaptation, the evolutionary process whereby over generations the members of a population become better suited to survive and reproduce in that environment. While negative selection decreases the prevalence of traits that diminish individuals' fitness, positive selection increases the prevalence of adaptive traits. Left untreated, SLE would have a reproductive fitness 
cost, defined as the ability to raise offspring that successfully reproduce. Thus, some evolutionary process must sustain the relative high frequency of SLE risk alleles seen in current populations around the world. We hypothesize that since the human genome is shaped by adaptation to environmental pressures at the population level, one plausible reason for the higher frequency of disease-risk alleles may be the direct effect of population-specific positive natural selection.

There is compelling evidence that natural selection is acting on a significant fraction of all genes $(\sim 3 \%)$ [4-7] and as much as $10 \%$ of the human genome [8]. Multiple studies have identified genes involved in immune-related functions to be under selection [8-10], including the HLA [11-14] (associated with all autoimmune diseases), BTLA [10] (associated with rheumatoid arthritis), ITPR3 [10] (SLE, type 1 diabetes, Grave's disease), PTPN22 [10] (rheumatoid arthritis, Crohn's disease, type 1 diabetes, vitiligo), ITGAX [10] (SLE), and BLK [10] (SLE, rheumatoid arthritis, Kawasaki disease). Finally, we have recently provided evidence that variants within the APOL1 gene known to be under selective pressure in some African populations predispose to end-stage kidney disease in SLE [15]. Given the increasing evidence of selection at loci associated with human autoimmune diseases, identification of alleles under selection may provide further insight into SLE susceptibility and help understand the natural history of SLE predisposition.

\section{Methods}

A list of genetic regions with compelling evidence of association with SLE was compiled from the literature. This list includes results that met genome-wide significance in any genome-wide association study (GWAS) or transethnic study of SLE and common or rare variants that are considered established SLE-predisposing loci from candidate gene and other studies. The list of regions was based on the literature as of August 2013 and comprises 89 genes in 74 genomic regions.

This list was built upon all the SLE-associated regions described in recent reviews [16-19], which include common and rare variants from candidate gene studies with compelling evidence of association with SLE. We included all reported risk variants for SLE using data from the National Human Genome Research Institute's Catalog of Published GWAS (http://www.genome.gov/gwastudies) accessed on August 30th, 2013 [20]. Finally, we searched PubMed (http:// www.ncbi.nlm.nih.gov/pubmed) for all large-scale transethnic or multiracial studies in SLE and catalogued all variants with a reported meta-analysis $P$ value $<5 \times 10^{-7}$. The references for these more recent studies are included in Table 1. Given the paucity of studies conducted in some minority populations, and in order to avoid differential bias due to the number of reported associations in different ethnic groups, we chose to include all variation regardless of the population(s) where they were reported and ignore the information about the population(s) where they have been reported to date.

Assuming no other influencing factors, the advantageous alleles at a locus under positive selective pressure will tend
TABLE 1: Genetic regions with compelling evidence for association with SLE.

\begin{tabular}{|c|c|c|}
\hline Gene(s) region & Chr & Pos $(\mathrm{Mb})$ \\
\hline $\mathrm{Clq}[21]$ & 1 & 22.96 \\
\hline IL12RB2 [22] & 1 & 67.55 \\
\hline PTPN22 [22-25] & 1 & 114.16 \\
\hline FCGR2A, FCGR3A $[26,27]$ & 1 & 159.74 \\
\hline TNFSF4 $[22,28-33]$ & 1 & 171.42 \\
\hline NMNAT2 $[22,24,32]$ & 1 & 181.48 \\
\hline NCF2 [22] & 1 & 181.79 \\
\hline APOBEC4 [28] & 1 & 181.88 \\
\hline CFH [34] & 1 & 194.89 \\
\hline CFHR1, CFHR4 [34] & 1 & 196.79 \\
\hline CRP [35] & 1 & 199.72 \\
\hline IL10 [22] & 1 & 205.01 \\
\hline LYST [22] & 1 & 233.89 \\
\hline RASGRP3 $[28,32]$ & 2 & 33.51 \\
\hline TET3, DGUOK [28] & 2 & 74.21 \\
\hline IFIH1 $[22,36]$ & 2 & 162.83 \\
\hline STAT4 $[22-24,32,37-41]$ & 2 & 191.60 \\
\hline PDCD1 [42] & 2 & 242.44 \\
\hline SCN10A [28] & 3 & 38.71 \\
\hline TREX1 [43] & 3 & 48.48 \\
\hline DNASE1L3 [44] & 3 & 58.15 \\
\hline PXK [22-24] & 3 & 58.29 \\
\hline TMEM39A [45] & 3 & 120.63 \\
\hline CD80 $[28]$ & 3 & 120.73 \\
\hline AFF1 [46] & 4 & 88.15 \\
\hline BANK1 $[23,28,47]$ & 4 & 102.93 \\
\hline LEF1 [46] & 4 & 109.19 \\
\hline IL21 [48] & 4 & 123.75 \\
\hline PPP2CA [49] & 5 & 133.53 \\
\hline TNIP1 $[22,28,32]$ & 5 & 150.39 \\
\hline PTTG1 $[22,32]$ & 5 & 159.78 \\
\hline $\mathrm{C} 4[50]$ & 6 & 32.09 \\
\hline HLA-DRB1 [24, 39, 51-53] & 6 & 32.59 \\
\hline ITPR3 [54] & 6 & 33.70 \\
\hline UHRF1BP1 [22, 28] & 6 & 34.87 \\
\hline $\mathrm{BACH} 2[28]$ & 6 & 90.69 \\
\hline ATG5, PRDM1 [22-24, 28] & 6 & 106.53 \\
\hline TNFAIP3 $[22,28,32,38,55]$ & 6 & 138.23 \\
\hline ICA1 $[22,24]$ & 7 & 8.12 \\
\hline JAZF1 [22] & 7 & 27.84 \\
\hline IKZF1 $[28,32]$ & 7 & 50.31 \\
\hline IRF5, TNPO3 [22, 24, 28, 39, 40, 56, 57] & 7 & 128.37 \\
\hline XKR6 $[24]$ & 8 & 10.79 \\
\hline BLK $[22-24,28,39,40]$ & 8 & 11.39 \\
\hline LYN [24] & 8 & 56.95 \\
\hline
\end{tabular}


TABLE 1: Continued.

\begin{tabular}{|c|c|c|}
\hline Gene(s) region & Chr & Pos $(\mathrm{Mb})$ \\
\hline ARMC3 [22] & 10 & 23.26 \\
\hline LRRC18, WDFY4 [28, 32, 33] & 10 & 49.89 \\
\hline ARID5B, RTKN2 [28] & 10 & 63.94 \\
\hline SLC29A3 [28] & 10 & 72.75 \\
\hline PHRF1, IRF7 [22-24] & 11 & 0.58 \\
\hline CD44, PDHX $[28,58]$ & 11 & 34.94 \\
\hline DDX6 $[22,32]$ & 11 & 118.13 \\
\hline ETS1 $[28,32,33]$ & 11 & 127.83 \\
\hline CREBL2, GPR19, CDKN1B [28] & 12 & 12.66 \\
\hline DRAM1 [28] & 12 & 102.27 \\
\hline SLC15A4 $[28,32]$ & 12 & 127.84 \\
\hline ELF1 [28] & 13 & 40.40 \\
\hline C2 [59] & 14 & 20.75 \\
\hline CSK [60] & 15 & 72.86 \\
\hline DNASE1 [61] & 16 & 3.64 \\
\hline CLEC16A [28] & 16 & 11.04 \\
\hline PRKCB [62] & 16 & 23.75 \\
\hline SEZ6L2 [28] & 16 & 29.79 \\
\hline ITGAM, ITGAX [23, 24, 28, 63] & 16 & 31.18 \\
\hline IRF8 $[22,45]$ & 16 & 84.49 \\
\hline IKZF3, ZPBP2 [45] & 17 & 37.91 \\
\hline CD226 $[22,57,64]$ & 18 & 65.68 \\
\hline TYK2 $[22,57]$ & 19 & 10.32 \\
\hline ICAM1, ICAM4, ICAM5 [65] & 19 & 10.40 \\
\hline ACP5 [66] & 19 & 11.55 \\
\hline DDA1 [28] & 19 & 17.28 \\
\hline UBE2L3 [22-24, 28] & 22 & 20.25 \\
\hline SCUBE1 [24] & 22 & 41.93 \\
\hline IRAK1, MECP2 $[22,23,67,68]$ & $\mathrm{X}$ & 152.93 \\
\hline
\end{tabular}

The reference list for each gene region does not intent to be exhaustive; instead, only the first and/or strongest associations reported to date are mentioned. A comprehensive list of all the studies that report each region have been recently reviewed elsewhere [16-18]. Chr: chromosome; Pos: position (in Mega basepairs) according to Human Genome Build hg18.

to stochastically increase in prevalence over generations. This can lead to allele frequency differences between populations, which can be detected using statistics that compare the genetic variability within and between populations [69]. It can also lead to the haplotype carrying the advantageous allele to remain longer than genetic distance predicts around alleles of equal frequency, which can be measured using haplotype-based statistics [7]. The evidence of selection in each SLE-associated region was analyzed using both population differentiation, allele frequency spectrum, and haplotype-based statistics in the HapMap II and HGDP populations as implemented in the Haplotter (http://haplotter. uchicago.edu/) [7] and the Human Genome Diversity Project (HGDP) Selection Browsers (http://hgdp.uchicago.edu/cgibin/gbrowse/HGDP/) [70], respectively.
Haplotter displays the results of a scan for positive selection in the human genome using the International HapMap Project data (http://haplotter.uchicago.edu/) [7]. These data consist of $\sim 800,000$ polymorphic SNPs in three distinct population samples of unrelated individuals: 89 Japanese and Han Chinese individuals from Tokyo and Beijing, respectively, denoted as East Asian (ASN), 60 individuals of northern and western European origin (CEU), and 60 Yoruba (YRI) from Ibadan, Nigeria. It shows results on the autosomes only. Results from several selection statistics are displayed, including (1) the fixation index $\left(F_{\mathrm{ST}}\right),(2)$ the Tajima's $D$, and (3) the integrated haplotype score (iHS). In situations where selection is restricted to certain populations or geographical locations, the allele frequencies at the locus that is undergoing selection may vary significantly between different populations. The fixation index $F_{\mathrm{ST}}$ provides a metric of the magnitude of global allele frequency differentiation between populations at a locus $[69,71] . F_{\mathrm{ST}}$ is directly related to the variance in allele frequency among populations and, conversely, to the degree of resemblance among individuals within populations. If $F_{\mathrm{ST}}$ is small, it means that the allele frequencies within each population are similar; if it is large, it means that the allele frequencies are different [72]. The Tajima's $D$ is based on the frequencies of the polymorphisms segregating in a locus [73]. As described [7], positive selection results in an excess of high frequency derived alleles compared to neutral expectations when the selected allele has swept to high frequencies. Positive selection also results in an excess of low frequency polymorphisms, especially when the selected allele is close to fixation or right after fixation. This skewing of SNP frequencies in different directions can be detected by Tajima's $D$, which is based on the frequencies of SNPs segregating in the region of interest [73]. Signals of selective sweeps will result in high negative $D$. The integrated haplotype score (iHS) uses the lengths of the haplotypes surrounding each core SNP to identify SNPs for which alleles have rapidly risen in frequency $[7,74]$. It is based on linkage disequilibrium (LD) surrounding a positively selected allele compared with background, providing evidence of recent positive selection at a locus [7]. An iHS score $>2.0$ reflects the fact that haplotypes on the ancestral background are longer compared with those on the derived allelic background.

For these analyses, genome-wide SNP data from Phase II of the HapMap Project were used to investigate if the regions associated with SLE showed evidence of selection in the CEU, YRI, and ASN populations using these three metrics (iHS, Tajima's $D$, and $F_{\mathrm{ST}}$ ). Regions of $1 \mathrm{Mb}$ around each of the 74 regions in Table 1 were queried, and, when higher than 2 , the maximum value on the $Y$-axis $(-\log (Q))$ in this $1 \mathrm{Mb}$ interval was recorded. As described by Voight et al. [7], the $-\log (Q)$ value represents the negative log of the rank of the observed statistic for a given SNP divided by the total number of SNPs. The statistic that is ranked is obtained independently for each of the three statistics separately for each population. For $D$, the estimated value of $D$ was used for ranking. For iHS, for each SNP, 25 SNPs on either side of the SNP are scanned for $|\mathrm{iHS}|>2$. The proportion of SNPs in this 51 SNP window with $|\mathrm{iHS}|>2$ is computed. For $F_{\mathrm{ST}}$, the statistic to be ranked is obtained in a similar manner as that for iHS 
except for each population comparison, the thresholds for defining a significant $F_{\mathrm{ST}}$ is based on the top 5\% cutoff for each population comparison. The different thresholds used for $F_{\mathrm{ST}}$ were CEU-YRI: 0.2976, CEU-ASN: 0.2055, and YRIASN: 0.3374. Haplotter also displays the $F_{\mathrm{ST}}$ value of the SNPs in the top $1 \%$ within each population comparison, which were also recorded, if any such SNPs were present in the $1 \mathrm{Mb}$ interval. In addition to these, Haplotter shows an empirical $P$ value estimated for each gene and for each population, as detailed by Voight et al. [7]. When this $P$ value showed significant evidence for selection, the value was recorded.

The HGDP Selection Browser displays results from a series of genome-wide scans for natural selection using single nucleotide polymorphism (SNP) genotype data from the Human Genome Diversity-CEPH Panel (HGDP), a dataset containing 938 individuals from 53 populations typed on the Illumina 650Y platform (http://hgdp.uchicago.edu/cgi-bin/ gbrowse/HGDP/) [70]. Summary statistics regarding haplotype structure and population differentiation on this data can be queried in the browser. These include the iHS, the $F_{\mathrm{ST}}$, and the cross-population extended haplotype homozygosity test (XP-EHH) [74]. While the iHS detects partial selective sweeps of moderate frequency ( 50\%-80\%), the XP-EHH detects selected alleles that have risen to near fixation in one population (above $80 \%$ frequency) [7,74]. As described by Pickrell et al. [70], the $F_{\mathrm{ST}}$ was calculated on the level of population groupings identified by Rosenberg et al. [75]; that is, if a SNP has high $F_{\mathrm{ST}}$, most of the variance in allele frequencies is captured by the seven labels identified in that paper. In the browser, plotted is the $-\log _{10}$ of the empirical $P$ value for each SNP-the higher this plotted $-\log _{10} P$ value, the more extreme (high) the $F_{\mathrm{ST}}$ value is compared the rest of the genotyped SNPs. The iHS was calculated as in Voight et al. [7] and smoothed across windows. Plotted is the $-\log _{10}$ of the $P$ value for a window centered at the SNP; high values again indicate potential signals of positive selection. The test statistic was the fraction of SNPs with $|\mathrm{iHS}|>2$. The XP-EHH was calculated as in Sabeti et al's work [74]. The test statistic was the maximum XP-EHH. Again, the plotted measure is a measure of how extreme a SNP is with regard to the rest of the genome, and high values indicate outliers potentially due to the action of natural selection. The iHS and XP-EHH have been calculated in each individual population, as well as in the following groupings: Bantu-speaking populations, Europeans, Middle Easterners, Central Asians, East Asians, Americans, and Oceanians.

Regions of $1 \mathrm{Mb}$ around each of the 74 regions in Table 1 were queried, and the maximum value on the $Y$-axis $(-\log (P))$ in this $1 \mathrm{Mb}$ interval was recorded.

\section{Results}

To test whether SLE susceptibility loci show evidence of positive selection, a list of 74 genetic regions with compelling evidence of association with SLE was compiled (Table 1). In order to test whether SLE-associated loci show evidence for recent positive selection, $1 \mathrm{Mb}$ regions around each of the 74 regions were queried. Regions where the maximum $-\log (Q)>3$ (for Haplotter) or $-\log (P)>3$ (for HGDP) for the $F_{\mathrm{ST}}, D$, iHS, or XP-EHH were considered as showing evidence for recent positive selection (Tables 2 and 3). In addition, regions that in the HapMap populations had SNPs with $F_{\mathrm{ST}}$ values in the top $1 \%$ within each population comparison, or whose empirical $P$ value estimated for each gene and for each population showed significant evidence for selection $(P$ value $<0.001)$ were also considered to show evidence for selection. Of the 74 regions associated with SLE, 19 showed evidence of selection in a HapMap population (Table 2), and 16 exhibited a signal of selection in a HGDP population (Table 3). Many of these loci also had corroborating evidence using different metrics.

In the HapMap data multiple regions displayed evidence of population differentiation, as indicated by the $F_{\mathrm{ST}}$, which was the highest in the PTPN22, TET3-DGUOK, ITPR3, ITGAM, and CD226 regions. Several SNPs with very high $F_{\mathrm{ST}}$ (in the top $1 \%$ within each population comparison) were identified in these and other regions, especially $X K R 6-B L K$ $\left(F_{\mathrm{ST}}=0.92\right.$ in YRI versus ASN $)$, TET3-DGUOK $\left(F_{\mathrm{ST}}=0.85\right.$ in YRI versus ASN, and $F_{\mathrm{ST}}=0.80$ in YRI versus CEU), CD226 $\left(F_{\mathrm{ST}}=0.80\right.$ in CEU versus YRI), LRRC18-WDFY4 $\left(F_{\mathrm{ST}}=0.80 \mathrm{in}\right.$ YRI versus ASN $), \operatorname{IFIH1}\left(F_{\mathrm{ST}}=0.78\right.$ in CEU versus YRI), PTPN22 ( $F_{\mathrm{ST}}=0.75$ in YRI versus ASN), and $\operatorname{ITGAM}\left(F_{\mathrm{ST}}=0.75\right.$ in YRI versus ASN). The highest allele frequency differences, as indicated by the $D$ statistic, were detected in the PTPN22, IFIH1, ITPR3, and XKR6-BLK regions. The ITPR 3 region also had a high iHS. This and $B L K$ are the regions that displayed the most consistently strong evidence for selection according to all three metrics. The ITPR 3 gene lies at $6 \mathrm{p} 21$, adjacent to the centromeric end of the extended MHC region, after the class II flanking region. XKR6 and $B L K$ lie on the same chromosomal inversion at 8p23.1. PTPN22, ITPR3, and CD226 exhibited the strongest evidence for selection according to the frequencybased statistics. Finally, several regions included genes whose empirical $P$ value showed significant evidence for selection. These genes included XKR6 $(P=0.004$ in ASN) and UHRF1BPI ( $P=0.006$ in CEU). Other genes were significant in several regions, such as the TET3-DGUOK region (DUSP11 and STAMBP with $P=0.005$ and $P=0.007$, resp., in CEU). The PTPN22, ITGAX (near ITGAM), ITPR3, and BLK regions were recently reported to be under selection (in YRI, YRI, YRI, and ASN, resp.) in a candidate gene study by Grossman et al. [10], who used full-genome sequence variation from the 1000 Genomes Project and the composite of multiple signals (CMS) test.

Since the regions in Table 2 showed evidence of selection in the HapMap samples, the evidence centered at the specific SNP associated with SLE were tested (Supplementary Table 1 in the Supplementary Material available online at http://dx.doi.org/10.1155/2014/203435). Specifically, Haplotter displays the iHS and $F_{\mathrm{ST}}$ for common SNPs. Of the queried SLE-associated SNPs, the highest evidence of population differentiation was shown by rs9937837 in ITGAM ( $F_{\text {ST }}=$ 0.81 in YRI versus ASN). Evidence for association according to the iHS test was observed in CFHR1-CFHR4 (rs16840639, $\mathrm{iHS}=-2.63 \mathrm{in} \mathrm{YRI})$, NMNAT2 $(\mathrm{rs} 2022013, \mathrm{iHS}=2.50 \mathrm{in}$ ASN), APOBEC4 (rs10911390, iHS $=-2.36$ in ASN), CFH 
TABle 2: Regions with evidence for selection on the HapMap populations.

\begin{tabular}{|c|c|c|c|c|c|c|c|c|c|c|c|}
\hline \multirow{2}{*}{ Gene region } & \multirow{2}{*}{$\mathrm{Chr}$} & \multirow{2}{*}{$\mathrm{Mb}$} & \multicolumn{2}{|l|}{ iHS } & \multicolumn{2}{|l|}{$D$} & \multicolumn{3}{|c|}{$F_{\mathrm{ST}}$} & \multicolumn{2}{|c|}{ Empirical $P$ value } \\
\hline & & & $\operatorname{Max}-\log (Q)$ & Pop & $\operatorname{Max}-\log (Q)$ & Pop & $\operatorname{Max}-\log (Q)$ & Value & Pop & Min $P$ value & Pop \\
\hline PTPN22 & 1 & 114.158 & - & - & 3.6 & YRI & 3.2 & 0.75 & YRI versus ASN & - & - \\
\hline TNFSF4 & 1 & 171.419 & 2.5 & ASN & 2.3 & ASN & 2.7 & 0.60 & YRI versus ASN & 0.005 & ASN \\
\hline NMNAT2 & 1 & 181.484 & 2.5 & ASN & 2.4 & CEU & - & - & - & 0.004 & ASN \\
\hline NCF2 & 1 & 181.791 & 2.5 & ASN & - & - & - & 0.65 & CEU versus YRI & 0.004 & ASN \\
\hline APOBEC4 & 1 & 181.882 & 2.5 & ASN & - & - & - & 0.65 & CEU versus YRI & 0.004 & ASN \\
\hline $\mathrm{CFH}$ & 1 & 194.888 & - & - & - & - & 3.0 & 0.60 & YRI versus ASN & - & - \\
\hline CFHR1, CFHR4 & 1 & 196.789 & 2.0 & YRI & - & - & 3.0 & 0.60 & YRI versus ASN & - & - \\
\hline TET3, DGUOK & 2 & 74.212 & 2.7 & $\mathrm{CEU}$ & 2.6 & ASN & 3.2 & 0.85 & YRI versus ASN & 0.001 & $\mathrm{CEU}$ \\
\hline IFIH1 & 2 & 162.832 & - & - & 3.8 & $\mathrm{CEU}$ & 2.2 & 0.78 & CEU versus YRI & - & - \\
\hline TREX1 & 3 & 48.481 & 2.4 & ASN & 2.1 & ASN & - & - & - & 0.002 & ASN \\
\hline TNIP1 & 5 & 150.390 & - & - & 3.0 & $\mathrm{CEU}$ & - & 0.65 & CEU versus YRI & - & - \\
\hline ITPR3 & 6 & 33.697 & 3.4 & YRI & 3.3 & YRI & 3.3 & 0.60 & YRI versus ASN & - & - \\
\hline UHRF1BP1 & 6 & 34.868 & 2.5 & $\mathrm{CEU}$ & 2.4 & YRI & - & 0.50 & - & 0.004 & $\mathrm{CEU}$ \\
\hline XKR6 & 8 & 10.791 & 2.7 & ASN & 3.3 & ASN & 2.6 & 0.92 & YRI versus ASN & 0.003 & ASN \\
\hline BLK & 8 & 11.389 & 2.7 & ASN & 3.2 & ASN & 2.6 & 0.92 & YRI versus ASN & 0.005 & ASN \\
\hline ARMC3 & 10 & 23.257 & - & - & 2.5 & $\mathrm{CEU}$ & 2.5 & 0.65 & YRI versus ASN & - & - \\
\hline LRRC18, WDFY4 & 10 & 49.893 & - & - & 2.0 & ASN & 2.5 & 0.80 & YRI versus ASN & - & - \\
\hline ITGAM & 16 & 31.179 & - & - & - & - & 3.4 & 0.75 & YRI versus ASN & - & - \\
\hline CD226 & 18 & 65.681 & - & - & 3.1 & $\mathrm{CEU}$ & 3.7 & 0.80 & CEU versus YRI & - & - \\
\hline
\end{tabular}

Regions were considered to show evidence for selection if the maximum $-\log (Q)>3$ for either the $F_{\mathrm{ST}}, D$, or iHS, or it had SNPs with $F_{\mathrm{ST}}$ values in the top $1 \%$ within each population comparison, or the empirical $P$ value estimated for the SLE-associated gene and for each population showed significant evidence for selection $(P$ value $<0.01)$. Cells that did not meet these thresholds or whose $-\log (Q)>2$ are marked with $(-)$. The table shows the highest $-\log (Q)$ value and respective population for the iHS, $D$, and $F_{\mathrm{ST}}$, the $F_{\mathrm{ST}}$ statistic (value) for SNPs in the top 1\% and the population comparison, and the minimum empirical $P$ value in each region. $Q$ is the rank of the observed statistic for a given SNP divided by the total number of SNPs. The statistic that is ranked is obtained independently for each of the three statistics separately for each population. For iHS, for each SNP, 25 SNPs on either side of the SNP are scanned for $\mid$ iHS $\mid>2$. The proportion of SNPs in this $51 \mathrm{SNP}$ window with $|\mathrm{iHS}|>2$ is computed. For $D$, the estimated value of $D$ was used for ranking. For $F_{\mathrm{ST}}$, the statistic to be ranked is obtained in a similar manner as that for iHS except for each population comparison, the thresholds for defining a significant $F_{\mathrm{ST}}$ is based on the top $5 \%$ cutoff for each population comparison. See Methods for details. Chr: chromosome, Mb: mega basepairs, Max: maximum, Min: minimum, Pop: population, ASN: East Asian, CEU: European, YRI: African.

TABLE 3: Regions with evidence for selection in the HGDP populations.

\begin{tabular}{|c|c|c|c|c|c|c|c|}
\hline \multirow{2}{*}{ Gene region } & \multirow{2}{*}{ Chr } & \multirow{2}{*}{$\mathrm{Mb}$} & \multirow{2}{*}{$\begin{array}{c}F_{\mathrm{ST}} \\
\operatorname{Max}-\log (P)\end{array}$} & \multicolumn{2}{|c|}{ iHS } & \multicolumn{2}{|c|}{ XP-EHH } \\
\hline & & & & $\operatorname{Max}-\log (P)$ & Pop & $\operatorname{Max}-\log (P)$ & Pop \\
\hline PTPN22 & 1 & 114.158 & 2.5 & 3 & Afr & 3.5 & Afr \\
\hline TNFSF4 & 1 & 171.419 & 4.5 & 2.5 & EAsia & 3.5 & EAsia \\
\hline CRP & 1 & 199.719 & 3.5 & - & - & 2.5 & Afr, Eur \\
\hline IL10 & 1 & 205.008 & 4 & 2 & MEast, EAsia & 2.5 & SAsia EAsia \\
\hline TET3, DGUOK & 2 & 74.212 & 2.5 & 2 & SAsia & 3.5 & MEast, SAsia \\
\hline TNIP1 & 5 & 150.390 & 3.5 & 1.5 & MEast & 3 & Amer \\
\hline PTTG1 & 5 & 159.781 & - & 3.5 & Afr & 2.8 & MEast, Afr \\
\hline UHRF1BP1 & 6 & 34.868 & - & 3 & Amer & 3.5 & Amer \\
\hline IKZF1 & 7 & 50.315 & 3.5 & 3 & EAsia & 2.5 & EAsia \\
\hline BLK & 8 & 11.389 & 4 & 3 & SAsia, MEast, Afr & 4 & EAsia \\
\hline ARMC3 & 10 & 23.257 & 2.5 & 2.5 & MEast & 3.5 & MEast \\
\hline SLC15A4 & 12 & 127.844 & 3.5 & - & - & 2.5 & Afr, Eur \\
\hline CLEC16A & 16 & 11.038 & 2 & 4 & Amer & 4 & Amer \\
\hline ITGAM & 16 & 31.179 & 2.5 & 2 & EAsia & 3.5 & EAsia \\
\hline IRF8 & 16 & 84.490 & 2.5 & 2 & SAsia & 4 & SAsia \\
\hline SCUBE1 & 22 & 41.929 & 2.5 & 2 & Oceania & 3 & Oceania \\
\hline
\end{tabular}

Regions were considered to show evidence for selection if the maximum $-\log _{10}(P)>3$ for either the $F_{\mathrm{ST}}$, iHS, or XP-EHH. The table shows the highest $-\log _{10}($ empirical $P$ value $)$ and respective population for the $F_{\mathrm{ST}}$, iHS, and XP-EHH in each region. Regions whose $-\log _{10}(P)<2$ are marked with $(-)$. See Methods for details. Chr: chromosome, Mb: mega basepairs, Max: maximum, Pop: population. Populations: Bantu-speaking Africans (Afr), Europeans (Eur), Middle Easterners (MEast), Eastern Asians (EAsia), South Asians (SAsia), Americans (Amer), and Oceanians (Oceania). 
TABLE 4: Summary of regions with evidence for selection on both the HapMap and HGDP populations.

\begin{tabular}{|c|c|c|c|c|c|c|c|c|}
\hline \multirow[b]{2}{*}{ Gene region } & \multicolumn{5}{|c|}{ HapMap } & \multicolumn{3}{|c|}{ HGDP } \\
\hline & iHS & $\begin{array}{c}D \\
\operatorname{Max}-\log (Q)\end{array}$ & $\begin{array}{r}F_{\mathrm{ST}} \\
\operatorname{Max}-\log (Q)\end{array}$ & Value & Min empirical $P$ value & $\begin{array}{c}F_{\mathrm{ST}} \\
\operatorname{Max}-\log (P)\end{array}$ & $\begin{array}{c}\text { iHS } \\
\operatorname{Max}-\log (P)\end{array}$ & $\begin{array}{c}\text { XP-EHH } \\
\text { Max }-\log (P) \\
\end{array}$ \\
\hline PTPN22 & & 3.6 & 3.2 & 0.75 & & & 3.0 & 3.5 \\
\hline TNFSF4 & & & & 0.6 & 0.005 & 4.5 & & \\
\hline TET3, DGUOK & & & 3.2 & 0.85 & 0.001 & & & 3.5 \\
\hline TNIP1 & & 3.0 & & 0.65 & & 3.5 & & 3.0 \\
\hline UHRF1BP1 & $|2.28|^{*}$ & & & & 0.004 & & 3.0 & 3.5 \\
\hline BLK & & & & 0.92 & 0.005 & 4.0 & 3.0 & 4.0 \\
\hline ITGAM & & & 3.4 & 0.75 & & & & 3.5 \\
\hline
\end{tabular}

Please refer to footnotes on Tables 2 and 3 for details. ${ }^{*}$ iHS $=-2.28$ for rs11755393.

(rs6677604, iHS $=-2.30$ in YRI), UHRF1BP1 (rs11755393, $\mathrm{iHS}=-2.28$ in CEU), and CD226 (rs727088, iHS $=2.14$ in CEU). The evidence for selection at the UHRF1BP1 variant was recently reported in a study of candidate inflammatorydisease SNPs using the same statistic and HapMap II data [76].

In the HGDP data, the highest XP-EHH was detected in the $B L K, C L E C 16 A$, and IRF8 regions and the maximum iHS in the CLEC16A and PTTG1 regions. The CLEC16A, BLK, $P T P N 22$, and $U H R F 1 B P 1$ regions showed strong evidence for selection under the haplotype-based statistics. TNFSF4, IL10, and $B L K$ were the regions showing the highest degree of population differentiation. The TNFSF 4 and BLK regions showed the strongest most consistent evidence of selection according to all three metrics. Using the same HapMap II data, Raj and colleagues [76] previously reported SNPs with a significant signal of selection in CLEC16A (rs12708716, iHS = 2.29 in CEU) and UHRF1BP1 (rs11755393, iHS $=-2.28$ in CEU). As mentioned, the BLK and ITGAX-ITGAM regions were recently reported to be under selection (in ASN and YRI, resp.) in a candidate genes study using the 1000 Genomes Project samples [10]. For the genes in Table 2, an inspection of the worldwide distribution of allele frequencies for the SNPs associated with SLE (Supplementary Table 2) revealed interesting patterns for SNPs in BLK, ITGAM, and CLEC16A (Figure 1).

Comparing the results of the tests for selection in the HapMap and the HGDP samples shows that there are seven genetic regions captured by at least one test in both datasets (Table 4). The common regions captured by the majority of tests were that of the PTPN22, UHRF1BP1, and BLK genes. While the region of the TNIP1 gene was captured in both the HapMap and HGDP populations by the frequency spectrum and population differentiation statistics $\left(D\right.$ and $\left.F_{\mathrm{ST}}\right)$, the region of the $U H R F 1 B P 1$ gene was captured by the haplotypebased statistics. The evidence for selection in these seven genetic regions (Table 4 ) is strengthened by the fact that they show consistent evidence across different studies and analytic methods.

\section{Discussion}

The diversity exhibited in the human genome is a result of stochastic population genetics processes such as mutation, migration, drift, and selection. SLE disproportionately affects women of child bearing age and without treatment would tend to put affected individuals at a reproductive disadvantage; here, reproductive disadvantage not only includes conception but the ability to raise offspring that successfully reproduce. Thus, strong alternative forces or changing selective pressure must exist that permits the relative high frequency of these risk alleles seen in current populations around the world. Infectious diseases and pathogenic exposures have been postulated to be important factors resulting in strong selective pressure and might provide such alternative pressures. This study investigated whether SLE susceptibility loci show signs of recent positive selection by comparing these regions to the background distribution of genetic variation.

Two important studies have computed several genomewide tests for selection in two main reference populations, the HapMap and the HGDP populations [7, 70], and implemented the results in genetic browsers. These browsers were queried to assess whether SLE-associated genetic regions have shown evidence for selection in the HapMap and HGDP populations.

This study reports several SLE-associated loci that show evidence for selection in the HapMap populations, and several SLE-associated loci that show evidence for selection in the HGDP populations. Seven genetic regions showed evidence for selection on both the HapMap and HGDP populations. These include the regions of the PTPN22, TNFSF4, TET3-DGUOK, TNIP1, UHRF1BP1, BLK, and ITGAM genes. In addition to the regions that are concordant, the different results obtained with the different metrics and datasets are expected, mostly due to the different coverage of the SNP arrays used, local adaptation in different ethnic groups, and the different test statistics which are likely recovering selective events from different time periods and for different stages of the selective sweep [77].

Several of these genes have been previously reported to show patterns of genetic variation that are consistent with evidence for recent positive selection. For example, in their search for inflammatory-disease SNPs that localize to regions of the genome where patterns of genetic variation are consistent with that expected under a model of recent positive selection, Raj and colleagues [76] also reported SNPs 


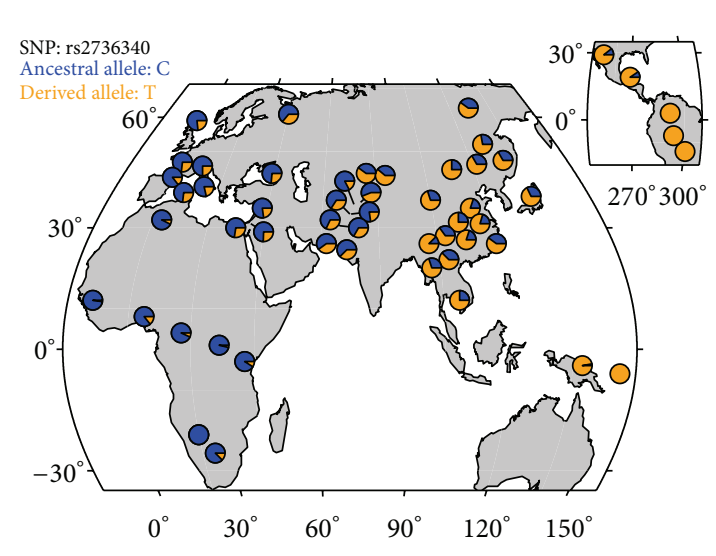

(a)

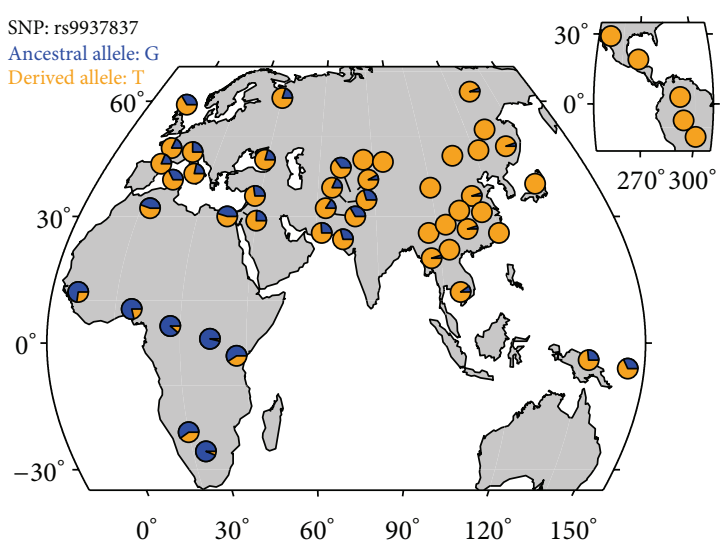

(b)

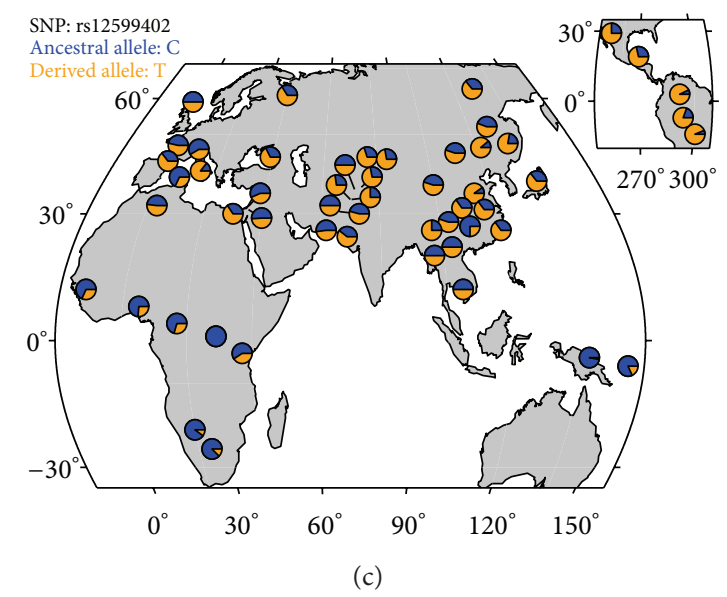

Figure 1: Worldwide distribution of allele frequencies for SLE-associated SNPs rs2736340 in BLK (a), rs9937837 in ITGAM (b), and rs12599402 in CLEC16A (c).

in CLEC16A and UHRF1BP1 that exhibit a significant signal of selection using the iHS test. Furthermore, they show that the SLE susceptibility allele in UHRF1BP1 is associated with decreased UHRF1BP1 RNA expression in different cell subsets, suggesting that the SLE risk allele is under recent selection and has a regulatory effect [76]. Furthermore, $U H R F 1 B P 1$ has been shown to be significantly differentially expressed in dendritic cells after Mycobacterium tuberculosis (MTB) infection [78]. Using full-genome sequence variation from the 1000 Genomes Project and the composite of multiple signals (CMS) test, Grossman et al. [10] reported the PTPN22, ITGAX (near ITGAM), ITPR3, and BLK regions to show evidence for recent positive selection.

Several of the immune genes that have been identified in regions under selection are under the selective pressure of known pathogens, such as the Duffy blood group atypical chemokine receptor $(D A R C)$ gene to Plasmodium vivax malaria [79], ras homolog family member A (RHOA), and OTU domain ubiquitin aldehyde binding 1 (OTUB1) genes to Yersinia pestis (plague) [80], or the tyrosylprotein sulfotransferase 1 (TPST1) gene to HIV [81]. Several genetic regions associated with susceptibility to different autoimmune diseases show evidence of selection that has been attributed to host-pathogen coevolution, including the multiple major histocompatibility complex (MHC) [82-84] and the celiac risk locus $\mathrm{SH} 2 \mathrm{~B} 3$ as a protective factor against bacterial infection [85]. Karlsson et al. [86] have recently reported that cholera has exerted strong selective pressure on proinflammatory pathways, and Jostins et al. [87] reported considerable overlap between susceptibility loci for inflammatory bowel disease and mycobacterial infection. Variants in the IFIH1 gene, whose protein is a cytoplasmic helicase that recognizes RNA of picornaviruses and mediates induction of interferon response to viral RNA, have been shown to affect IFIH1 function and host antiviral response [88]. In the context of SLE predisposing loci, Clatworthy et al. [89] have shown that FCGR2B is important in controlling the immune response to Plasmodium falciparum, the parasite responsible for the most severe form of malaria, and suggests that the higher frequency of human FCGR2B polymorphisms predisposing to SLE in Asians and Africans may be maintained because these variants reduce susceptibility to malaria. The complement component $(3 \mathrm{~b} / 4 \mathrm{~b})$ receptor 1 (CR1) gene has been shown to be a $P$. falciparum resistance gene [90] used by the parasite for host invasion. Machado et al. [91] have suggested that helminth infection has driven positive selection of FCGRs variation. Finally, Grossman et al. [10] implicated Salmonella typhimurium and other exposures that directionally drive 
selection of the toll-like receptor 5 (TLR5) gene [92]. Given that infectious organisms are strong agents of natural selection, it is plausible that alleles selected for protection against infection predispose to autoimmune diseases.

It is important to acknowledge the challenges and limitations inherent to the study of traits with complex genetic architectures and/or a less clear influence on survival and reproduction, such as SLE. As Castiblanco and colleagues [93] recently articulated, the differences in allele and genotype frequencies of diverse human populations depend upon their evolutionary and epidemiological history, including environmental exposures, which might explain why some risk alleles to autoimmunity may be protective factors to infectious diseases and vice versa in a given population (e.g., PTPN22 [94, 95] and TNF [96]). Immune and infectious agents have been recognized as among the strongest selective pressures for natural populations, as shown by the identification of candidate adaptive alleles that functionally contribute to biological variation in contemporary populations. However, clarifying the relationship between the functional alleles and reproductive fitness in the environment in which they rose to a high frequency in the ancestors of the study population can rarely be attained. In complex diseases such as SLE, despite the established associations to specific regions or polymorphisms, the true causal variants still remain largely unknown. The emerging availability of genome-wide functional data allows the integration of an unprecedented amount of biological information to help identify potential functional variants and characterize their biological impact. Recent examples demonstrate how the integration of signatures of positive selection with phenotypic association studies and/or with regulatory data can improve the identification of functional loci [10, 97-99]. Also, the complex genetic architecture of SLE, resulting from the effects of many alleles of small effects, suggests that adaptation is likely to have occurred by simultaneous selection on variants at many loci. In this scenario, the response to selection is due to small frequency shifts of many alleles. However, most methods to detect selection rely on rapid fixation of strongly selected alleles. The development of novel analytical approaches to detect more subtle signatures of selection will improve the identification of selection signatures in complex diseases like SLE. Clearly, much remains to be done until the functional adaptive SLE risk loci are identified, the phenotypic consequences of these risk alleles elucidated, and the relationship between the functional alleles and reproductive fitness clarified. Recent progresses will provide the necessary tools to accelerate the discovery of these functional adaptive variants that increase the risk of SLE, which will improve knowledge about the etiology and deepen our understanding of the natural history of SLE. Further research regarding exploration of the interplay between infection, type of exposure, additional environmental factors, and autoimmunity will result in the discovery of multiple factors underpinning perhaps newly identified physiopathology mechanisms of SLE and autoimmune diseases [93].

In summary, this study has systematically queried the HapMap and HGDP populations for evidence for selection at SLE susceptibility regions and provides a comprehensive catalog of regions with both evidence for recent positive selection and association with SLE. These results provide support for recent positive selection influencing genetic variation associated with SLE, suggesting that populationspecific selective pressures may be one of the factors behind the high frequency of SLE risk alleles in the population and differential disease risk. Finally, these results support future analyses aimed at identifying the specific selective pressures and characterizing the functional mechanisms of adaptation and disease predisposition.

\section{Conflict of Interests}

The authors declare that there is no conflict of interests regarding the publication of this paper.

\section{Acknowledgments}

The authors would like to thank Mia T. Chandler for assistance in compiling the list with SLE-associated regions. This study was supported by the US National Institutes of Health (NIH) Grant P60 AR062755, by the South Carolina Clinical \& Translational Research (SCTR) Institute, with an academic home at the Medical University of South Carolina, through NIH Grants nos. UL1 RR029882 and UL1 TR000062, and by the WFU Center for Public Health Genomics.

\section{References}

[1] C. A. Peschken, S. J. Katz, E. Silverman et al., "The 1000 Canadian faces of lupus: determinants of disease outcome in a large multiethnic cohort," Journal of Rheumatology, vol. 36, no. 6, pp. 1200-1208, 2009.

[2] M. Fernández, G. S. Alarcón, J. Calvo-Alén et al., "A multiethnic, multicenter cohort of patients with Systemic Lupus Erythematosus (SLE) as a model for the study of ethnic disparities in SLE," Arthritis Care and Research, vol. 57, no. 4, pp. 576-584, 2007.

[3] D. P. Strachan, "Hay fever, hygiene, and household size," British Medical Journal, vol. 299, no. 6710, pp. 1259-1260, 1989.

[4] M. A. Eberle, M. J. Rieder, L. Kruglyak, and D. A. Nickerson, "Allele frequency matching between SNPs reveals an excess of linkage disequilibrium in genic regions of the human genome," PLoS Genetics, vol. 2, no. 9, article el42, 2006.

[5] P. C. Sabeti, D. E. Reich, J. M. Higgins et al., "Detecting recent positive selection in the human genome from haplotype structure," Nature, vol. 419, no. 6909, pp. 832-837, 2002.

[6] J. M. Smith and J. Haigh, "The hitch hiking effect of a favourable gene," Genetical Research, vol. 23, no. 1, pp. 23-35, 1974.

[7] B. F. Voight, S. Kudaravalli, X. Wen, and J. K. Pritchard, "A map of recent positive selection in the human genome," PLoS Biology, vol. 4, no. 3, article e72, 2006.

[8] S. H. Williamson, M. J. Hubisz, A. G. Clark, B. A. Payseur, C. D. Bustamante, and R. Nielsen, "Localizing recent adaptive evolution in the human genome," PLoS Genetics, vol. 3, no. 6, article e90, 2007.

[9] M. Fumagalli, R. Cagliani, U. Pozzoli et al., "Widespread balancing selection and pathogen-driven selection at blood group antigen genes," Genome Research, vol. 19, no. 2, pp. 199212, 2009. 
[10] S. R. Grossman, K. G. Andersen, I. Shlyakhter et al., "Identifying recent adaptations in large-scale genomic data," Cell, vol. 152, pp. 703-713, 2013.

[11] F. L. Black and P. W. Hedrick, "Strong balancing selection at HLA loci: evidence from segregation in South Amerindian families," Proceedings of the National Academy of Sciences of the United States of America, vol. 94, no. 23, pp. 12452-12456, 1997.

[12] R. Cagliani, S. Riva, U. Pozzoli et al., "Balancing selection is common in the extended MHC region but most alleles with opposite risk profile for autoimmune diseases are neutrally evolving," BMC Evolutionary Biology, vol. 11, no. 1, article 171, 2011.

[13] X. Liu, Y. Fu, Z. Liu et al., "An ancient balanced polymorphism in a regulatory region of human major histocompatibility complex is retained in Chinese minorities but lost worldwide," American Journal of Human Genetics, vol. 78, no. 3, pp. 393-400, 2006.

[14] Z. Tan, A. M. Shon, and C. Ober, "Evidence of balancing selection at the HLA-G promoter region," Human Molecular Genetics, vol. 14, no. 23, pp. 3619-3628, 2005.

[15] B. I. Freedman, C. D. Langefeld, K. K. Andringa et al., "Endstage kidney disease in African Americans with lupus nephritis associates with APOL1," Arthritis and Rheumatism, 2013.

[16] O. J. Rullo and B. P. Tsao, "Recent insights into the genetic basis of systemic lupus erythematosus," Annals of the Rheumatic Diseases, vol. 72, Suppl 2, pp. ii56-ii61, 2013.

[17] S. E. Vaughn, L. C. Kottyan, M. E. Munroe, and J. B. Harley, "Genetic susceptibility to lupus: the biological basis of genetic risk found in B cell signaling pathways," Journal of Leukocyte Biology, vol. 92, pp. 577-591, 2012.

[18] S. G. Guerra, T. J. Vyse, and D. S. Cunninghame Graham, "The genetics of lupus: a functional perspective," Arthritis Research \& Therapy, vol. 14, no. 3, article 211, 2012.

[19] P. S. Ramos, E. E. Brown, R. P. Kimberly, and C. D. Langefeld, "Genetic factors predisposing to systemic lupus erythematosus and lupus nephritis," Seminars in Nephrology, vol. 30, no. 2, pp. 164-176, 2010.

[20] L. A. Hindorff, H. Junkins, J. P. Mehta, and T. A. Manolio, "A catalog of published genome-wide association studies," 2010, http://www.genome.gov/gwastudies/ .

[21] H. Nishino, K. Shibuya, Y. Nishida, and M. Mushimoto, "Lupus erythematosus-like syndrome with selective complete deficiency of C1q," Annals of Internal Medicine, vol. 95, no. 3, pp. 322-324, 1981.

[22] V. Gateva, J. K. Sandling, G. Hom et al., "A large-scale replication study identifies TNIP1, PRDM1, JAZF1, UHRF1BP1 and IL10 as risk loci for systemic lupus erythematosus," Nature Genetics, vol. 41, no. 11, pp. 1228-1233, 2009.

[23] R. R. Graham, G. Hom, W. Ortmann, and T. W. Behrens, "Review of recent genome-wide association scans in lupus," Journal of Internal Medicine, vol. 265, no. 6, pp. 680-688, 2009.

[24] J. B. Harley, M. E. Alarcón-Riquelme, L. A. Criswell et al., "Genome-wide association scan in women with systemic lupus erythematosus identifies susceptibility variants in ITGAM, PXK, KIAA1542 and other loci," Nature Genetics, vol. 40, no. 2, pp. 204-210, 2008.

[25] C. Kyogoku, C. D. Langefeld, W. A. Ortmann et al., "Genetic association of the R620W polymorphism of protein tyrosine phosphatase PTPN22 with human SLE," American Journal of Human Genetics, vol. 75, no. 3, pp. 504-507, 2004.
[26] F. B. Karassa, T. A. Trikalinos, and J. P. A. Ioannidis, "Role of the $\mathrm{F} c \gamma$ receptor IIa polymorphism in susceptibility to systemic lupus erythematosus and lupus nephritis: a meta-analysis," Arthritis and Rheumatism, vol. 46, no. 6, pp. 1563-1571, 2002.

[27] F. B. Karassa, T. A. Trikalinos, J. P. A. Ioannidis et al., "The Fc $\gamma$ RIIIA-F158 allele is a risk factor for the development of lupus nephritis: a meta-analysis," Kidney International, vol. 63, no. 4, pp. 1475-1482, 2003.

[28] W. Yang, H. Tang, Y. Zhang et al., "Meta-analysis followed by replication identifies loci in or near CDKN1B, TET3, CD80, DRAM1, and ARID5B as associated with systemic lupus erythematosus in Asians," American Journal of Human Genetics, vol. 92, no. 1, pp. 41-51, 2013.

[29] Y. K. Chang, W. Yang, M. Zhao et al., "Association of BANK1 and TNFSF4 with systemic lupus erythematosus in Hong Kong Chinese," Genes and Immunity, vol. 10, no. 5, pp. 414-420, 2009.

[30] D. S. C. Graham, R. R. Graham, H. Manku et al., "Polymorphism at the TNF superfamily gene TNFSF4 confers susceptibility to systemic lupus erythematosus," Nature Genetics, vol. 40, no. 1, pp. 83-89, 2008.

[31] A. M. Delgado-Vega, A. K. Abelson, E. Sánchez et al., "Replication of the TNFSF4 (OX40L) promoter region association with systemic lupus erythematosus," Genes and Immunity, vol. 10, no. 3, pp. 248-253, 2009.

[32] J. W. Han, H. F. Zheng, Y. Cui et al., "Genome-wide association study in a Chinese Han population identifies nine new susceptibility loci for systemic lupus erythematosus," Nature Genetics, vol. 41, no. 11, pp. 1234-1237, 2009.

[33] W. Yang, N. Shen, D. Q. Ye et al., "Genome-wide association study in asian populations identifies variants in ETS1 and WDFY4 associated with systemic lupus erythematosus," PLoS Genetics, vol. 6, no. 2, Article ID e1000841, 2010.

[34] J. Zhao, H. Wu, M. Khosravi et al., "Association of genetic variants in complement factor $\mathrm{H}$ and factor $\mathrm{H}$-related genes with systemic lupus erythematosus susceptibility," PLoS Genetics, vol. 7, no. 5, Article ID e1002079, 2011.

[35] J. C. Edberg, J. Wu, C. D. Langefeld et al., "Genetic variation in the CRP promoter: association with systemic lupus erythematosus," Human Molecular Genetics, vol. 17, no. 8, pp. 1147$1155,2008$.

[36] J. E. Molineros, A. K. Maiti, C. Sun et al., "Admixture mapping in lupus identifies multiple functional variants within IFIH1 associated with apoptosis, inflammation, and autoantibody production," PLoS Genetics, vol. 9, Article ID e1003222, 2013.

[37] A. K. Abelson, A. M. Delgado-Vega, S. V. Kozyrev et al., "STAT4 associates with systemic lupus erythematosus through two independent effects that correlate with gene expression and act additively with IRF5 to increase risk," Annals of the Rheumatic Diseases, vol. 68, no. 11, pp. 1746-1753, 2009.

[38] R. R. Graham, C. Cotsapas, L. Davies et al., "Genetic variants near TNFAIP3 on 6q23 are associated with systemic lupus erythematosus," Nature Genetics, vol. 40, no. 9, pp. 1059-1061, 2008.

[39] G. Hom, R. R. Graham, B. Modrek et al., "Association of systemic lupus erythematosus with C8orf13-BLK and ITGAMITGAX," New England Journal of Medicine, vol. 358, no. 9, pp. 900-909, 2008.

[40] Y. H. Lee, S. C. Bae, S. J. Choi, J. D. Ji, and G. G. Song, "Genomewide pathway analysis of genome-wide association studies on systemic lupus erythematosus and rheumatoid arthritis," Molecular Biology Reports, vol. 39, pp. 10627-10635, 2012. 
[41] E. F. Remmers, R. M. Plenge, A. T. Lee et al., "STAT4 and the risk of rheumatoid arthritis and systemic lupus erythematosus," New England Journal of Medicine, vol. 357, no. 10, pp. 977-986, 2007.

[42] L. Prokunina, C. Castillejo-López, F. Öberg et al., "A regulatory polymorphism in PDCD1 is associated with susceptibility to systemic lupus erythematosus in humans," Nature Genetics, vol. 32, no. 4, pp. 666-669, 2002.

[43] M. A. Lee-Kirsch, M. Gong, D. Chowdhury et al., "Mutations in the gene encoding the 3/-5/ DNA exonuclease TREX1 are associated with systemic lupus erythematosus," Nature Genetics, vol. 39, no. 9, pp. 1065-1067, 2007.

[44] S. M. Al-Mayouf, A. Sunker, R. Abdwani et al., "Loss-offunction variant in DNASE1L3 causes a familial form of systemic lupus erythematosus," Nature Genetics, vol. 43, no. 12, pp. 1186-1188, 2011.

[45] C. J. Lessard, I. Adrianto, J. A. Ice et al., "Identification of IRF8, TMEM39A, and IKZF3-ZPBP2 as susceptibility loci for systemic lupus erythematosus in a large-scale multiracial replication study," American Journal of Human Genetics, vol. 90, no. 4, pp. 648-660, 2012.

[46] Y. Okada, K. Shimane, Y. Kochi et al., "A genome-wide association study identified AFF1 as a susceptibility locus for systemic lupus eyrthematosus in Japanese," PLoS Genetics, vol. 8, no. 1, Article ID e1002455, 2012.

[47] S. V. Kozyrev, A. K. Abelson, J. Wojcik et al., "Functional variants in the B-cell gene BANK1 are associated with systemic lupus erythematosus," Nature Genetics, vol. 40, no. 2, pp. 211216, 2008.

[48] T. Hughes, X. Kim-Howard, J. A. Kelly et al., "Fine-mapping and transethnic genotyping establish IL2/IL21 genetic association with lupus and localize this genetic effect to IL21," Arthritis and Rheumatism, vol. 63, no. 6, pp. 1689-1697, 2011.

[49] W. Tan, K. Sunahori, J. Zhao et al., "Association of PPP2CA polymorphisms with systemic lupus erythematosus susceptibility in multiple ethnic groups," Arthritis and Rheumatism, vol. 63, no. 9, pp. 2755-2763, 2011.

[50] L. Boteva, D. L. Morris, J. Cortés-Hernández, J. Martin, T. J. Vyse, and M. M. A. Fernando, "Genetically determined partial complement $\mathrm{C} 4$ deficiency states are not independent risk factors for SLE in UK and Spanish populations," American Journal of Human Genetics, vol. 90, no. 3, pp. 445-456, 2012.

[51] M. M. A. Fernando, C. R. Stevens, P. C. Sabeti et al., "Identification of two independent risk factors for lupus within the MHC in United Kingdom families," PLoS Genetics, vol. 3, no. 11, article e192, 2007.

[52] F. C. Grumet, A. Coukell, J. G. Bodmer, W. F. Bodmer, and H. O. McDevitt, "Histocompatibility (HL-A) antigens associated with systemic lupus erythematosus. A possible genetic predisposition to disease," New England Journal of Medicine, vol. 285, no. 4, pp. 193-196, 1971.

[53] H. Waters, P. Konrad, and R. L. Walford, "The distribution of HL-A histocompatibility factors and genes in patients with systemic lupus erythematosus," Tissue Antigens, vol. 1, no. 2, pp. 68-73, 1971.

[54] T. Oishi, A. Iida, S. Otsubo et al., "A functional SNP in the NKX2.5-binding site of ITPR3 promoter is associated with susceptibility to systemic lupus erythematosus in Japanese population," Journal of Human Genetics, vol. 53, no. 2, pp. 151$162,2008$.

[55] S. L. Musone, K. E. Taylor, T. T. Lu et al., "Multiple polymorphisms in the TNFAIP3 region are independently associated with systemic lupus erythematosus," Nature Genetics, vol. 40, no. 9, pp. 1062-1064, 2008.

[56] R. R. Graham, S. V. Kozyrev, E. C. Baechler et al., "A common haplotype of interferon regulatory factor 5 (IRF5) regulates splicing and expression and is associated with increased risk of systemic lupus erythematosus," Nature Genetics, vol. 38, no. 5, pp. 550-555, 2006.

[57] S. Sigurdsson, G. Nordmark, H. H. H. Göring et al., "Polymorphisms in the tyrosine kinase 2 and interferon regulatory factor 5 genes are associated with systemic lupus erythematosus," American Journal of Human Genetics, vol. 76, no. 3, pp. 528-537, 2005.

[58] C. J. Lessard, I. Adrianto, J. A. Kelly et al., "Identification of a systemic lupus erythematosus susceptibility locus at $11 \mathrm{p} 13$ between PDHX and CD44 in a multiethnic study," American Journal of Human Genetics, vol. 88, no. 1, pp. 83-91, 2011.

[59] V. Agnello, M. M. De Bracco, and H. G. Kunkel, "Hereditary C2 deficiency with some manifestations of systemic lupus erythematosus," Journal of Immunology, vol. 108, no. 3, pp. 837840, 1972.

[60] N. Manjarrez-Orduno, E. Marasco, S. A. Chung et al., "CSK regulatory polymorphism is associated with systemic lupus erythematosus and influences B-cell signaling and activation," Nature Genetics, vol. 44, pp. 1227-1230, 2012.

[61] K. Yasutomo, T. Horiuchi, S. Kagami et al., "Mutation of DNASE1 in people with systemic lupus erythematosus," Nature Genetics, vol. 28, no. 4, pp. 313-314, 2001.

[62] Y. J. Sheng, J. P. Gao, J. Li et al., "Follow-up study identifies two novel susceptibility loci PRKCB and 8p11.21 for systemic lupus erythematosus," Rheumatology, vol. 50, no. 4, pp. 682-688, 2011.

[63] S. K. Nath, S. Han, X. Kim-Howard et al., "A nonsynonymous functional variant in integrin- $\alpha \mathrm{M}$ (encoded by ITGAM) is associated with systemic lupus erythematosus," Nature Genetics, vol. 40, no. 2, pp. 152-154, 2008.

[64] S. E. Löfgren, A. M. Delgado-Vega, C. J. Gallant et al., "A 3/-untranslated region variant is associated with impaired expression of CD226 in T and natural killer T cells and is associated with susceptibility to systemic lupus erythematosus," Arthritis and Rheumatism, vol. 62, no. 11, pp. 3404-3414, 2010.

[65] K. Kim, E. E. Brown, C. B. Choi et al., "Variation in the ICAM1-ICAM4-ICAM5 locus is associated with systemic lupus erythematosus susceptibility in multiple ancestries," Annals of the Rheumatic Diseases, vol. 71, pp. 1809-1814, 2012.

[66] T. A. Briggs, G. I. Rice, S. Daly et al., "Tartrate-resistant acid phosphatase deficiency causes a bone dysplasia with autoimmunity and a type i interferon expression signature," Nature Genetics, vol. 43, no. 2, pp. 127-131, 2011.

[67] C. O. Jacob, J. Zhu, D. L. Armstrong et al., "Identification of IRAK1 as a risk gene with critical role in the pathogenesis of systemic lupus erythematosus," Proceedings of the National Academy of Sciences of the United States of America, vol. 106, no. 15, pp. 6256-6261, 2009.

[68] R. Webb, J. D. Wren, M. Jeffries et al., "Variants within MECP2, a key transcription regulator, are associated with increased susceptibility to lupus and differential gene expression in patients with systemic lupus erythematosus," Arthritis and Rheumatism, vol. 60, no. 4, pp. 1076-1084, 2009.

[69] R. C. Lewontin and J. Krakauer, "Distribution of gene frequency as a test of the theory of the selective neutrality of polymorphisms," Genetics, vol. 74, no. 1, pp. 175-195, 1973. 
[70] J. K. Pickrell, G. Coop, J. Novembre et al., "Signals of recent positive selection in a worldwide sample of human populations," Genome Research, vol. 19, no. 5, pp. 826-837, 2009.

[71] C. C. Cockerham and B. S. Weir, "Estimation of inbreeding parameters in stratified populations," Annals of Human Genetics, vol. 50, no. 3, pp. 271-281, 1986.

[72] K. E. Holsinger and B. S. Weir, "Genetics in geographically structured populations: defining, estimating and interpreting FST," Nature Reviews Genetics, vol. 10, no. 9, pp. 639-650, 2009.

[73] F. Tajima, "Statistical method for testing the neutral mutation hypothesis by DNA polymorphism," Genetics, vol. 123, no. 3, pp. 585-595, 1989.

[74] P. C. Sabeti, P. Varilly, B. Fry et al., "Genome-wide detection and characterization of positive selection in human populations," Nature, vol. 449, pp. 913-918, 2007.

[75] N. A. Rosenberg, J. K. Pritchard, J. L. Weber et al., "Genetic structure of human populations," Science, vol. 298, no. 5602, pp. 2381-2385, 2002.

[76] T. Raj, M. Kuchroo, J. M. Replogle et al., "Common risk alleles for inflammatory diseases are targets of recent positive selection," American Journal of Human Genetics, vol. 92, pp. 517529, 2013

[77] J. M. Akey, "Constructing genomic maps of positive selection in humans: where do we go from here?" Genome Research, vol. 19, no. 5, pp. 711-722, 2009.

[78] L. B. Barreiro, L. Tailleux, A. A. Pai, B. Gicquel, J. C. Marioni, and Y. Gilad, "Deciphering the genetic architecture of variation in the immune response to Mycobacterium tuberculosis infection," Proceedings of the National Academy of Sciences of the United States of America, vol. 109, no. 4, pp. 1204-1209, 2012.

[79] P. C. Sabeti, S. F. Schaffner, B. Fry et al., "Positive natural selection in the human lineage," Science, vol. 312, no. 5780, pp. 1614-1620, 2006.

[80] M. J. Edelmann, H. B. Kramer, M. Altun, and B. M. Kessler, "Post-translational modification of the deubiquitinating enzyme otubain 1 modulates active RhoA levels and susceptibility to Yersinia invasion," FEBS Journal, vol. 277, no. 11, pp. 2515-2530, 2010.

[81] M. Farzan, T. Mirzabekov, P. Kolchinsky et al., "Tyrosine sulfation of the amino terminus of CCR5 facilitates HIV-1 entry," Cell, vol. 96, no. 5, pp. 667-676, 1999.

[82] A. L. Hughes and M. Nei, "Pattern of nucleotide substitution at major histocompatibility complex class I loci reveals overdominant selection," Nature, vol. 335, no. 6186, pp. 167-170, 1988.

[83] F. Prugnolle, A. Manica, M. Charpentier, J. F. Guégan, V. Guernier, and F. Balloux, "Pathogen-driven selection and worldwide HLA class I diversity," Current Biology, vol. 15, no. 11, pp. 10221027, 2005.

[84] N. Qutob, F. Balloux, T. Raj et al., "Signatures of historical demography and pathogen richness on MHC class i genes," Immunogenetics, vol. 64, no. 3, pp. 165-175, 2012.

[85] A. Zhernakova, C. C. Elbers, B. Ferwerda et al., "Evolutionary and functional analysis of celiac risk loci reveals SH2B3 as a protective factor against bacterial infection," American Journal of Human Genetics, vol. 86, no. 6, pp. 970-977, 2010.

[86] E. K. Karlsson, J. B. Harris, S. Tabrizi et al., "Natural selection in a bangladeshi population from the cholera-endemic ganges river delta," Science Translational Medicine, vol. 5, no. 192, Article ID 192ra186, 2013.

[87] L. Jostins, S. Ripke, R. K. Weersma et al., "Host-microbe interactions have shaped the genetic architecture of inflammatory bowel disease," Nature, vol. 491, pp. 119-124, 2012.
[88] S. Nejentsev, N. Walker, D. Riches, M. Egholm, and J. A. Todd, "Rare variants of IFIH1, a gene implicated in antiviral responses, protect against type 1 diabetes," Science, vol. 324, no. 5925, pp. 387-389, 2009.

[89] M. R. Clatworthy, L. Willcocks, B. Urban et al., "Systemic lupus erythematosus-associated defects in the inhibitory receptor Fc $\gamma$ RIIb reduce susceptibility to malaria," Proceedings of the National Academy of Sciences of the United States of America, vol. 104, no. 17, pp. 7169-7174, 2007.

[90] I. A. Cockburn, M. J. Mackinnon, A. O’Donnell et al., “A human complement receptor 1 polymorphism that reduces Plasmodium falciparum rosetting confers protection against severe malaria," Proceedings of the National Academy of Sciences of the United States of America, vol. 101, no. 1, pp. 272-277, 2004.

[91] L. R. Machado, R. J. Hardwick, J. Bowdrey et al., "Evolutionary history of copy-number-variable locus for the low-affinity Fcgamma receptor: mutation rate, autoimmune disease, and the legacy of helminth infection," American Journal of Human Genetics, vol. 90, pp. 973-985, 2012.

[92] T. R. Hawn, H. Wu, J. M. Grossman, B. H. Hahn, B. P. Tsao, and A. Aderem, "A stop codon polymorphism of Toll-like receptor 5 is associated with resistance to systemic lupus erythematosus," Proceedings of the National Academy of Sciences of the United States of America, vol. 102, no. 30, pp. 10593-10597, 2005.

[93] J. Castiblanco, M. Arcos-Burgos, and J. M. Anaya, "What is next after the genes for autoimmunity?” BMC Medicine, vol. 11, article 197, 2013.

[94] L. M. Gomez, J. M. Anaya, C. I. Gonzalez et al., "PTPN22 C1858T polymorphism in Colombian patients with autoimmune diseases," Genes and Immunity, vol. 6, no. 7, pp. 628-631, 2005.

[95] L. M. Gomez, J. M. Anaya, and J. Martin, "Genetic Influence of PTPN22 R620W Polymorphism in Tuberculosis," Human Immunology, vol. 66, no. 12, pp. 1242-1247, 2005.

[96] P. A. Correa, L. M. Gomez, J. Cadena, and J. M. Anaya, "Autoimmunity and tuberculosis. Opposite association with TNF polymorphism," Journal of Rheumatology, vol. 32, no. 2, pp. 219-224, 2005.

[97] B. Vernot, A. B. Stergachis, M. T. Maurano et al., "Personal and population genomics of human regulatory variation," Genome Research, vol. 22, pp. 1689-1697, 2012.

[98] S. Kudaravalli, J. B. Veyrieras, B. E. Stranger, E. T. Dermitzakis, and J. K. Pritchard, "Gene expression levels are a target of recent natural selection in the human genome," Molecular Biology and Evolution, vol. 26, no. 3, pp. 649-658, 2009.

[99] H. B. Fraser, "Gene expression drives local adaptation in humans," Genome Research, vol. 23, pp. 1089-1096, 2013. 


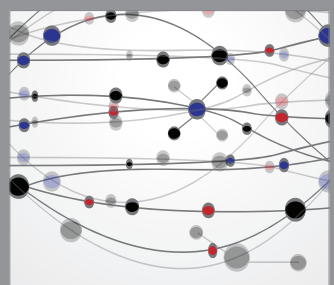

The Scientific World Journal
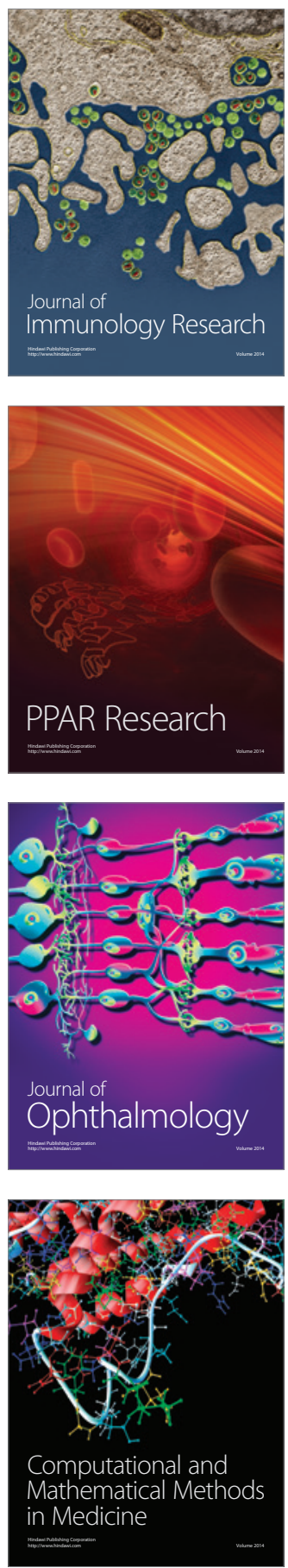

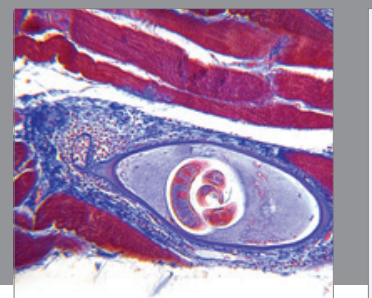

Gastroenterology

Research and Practice
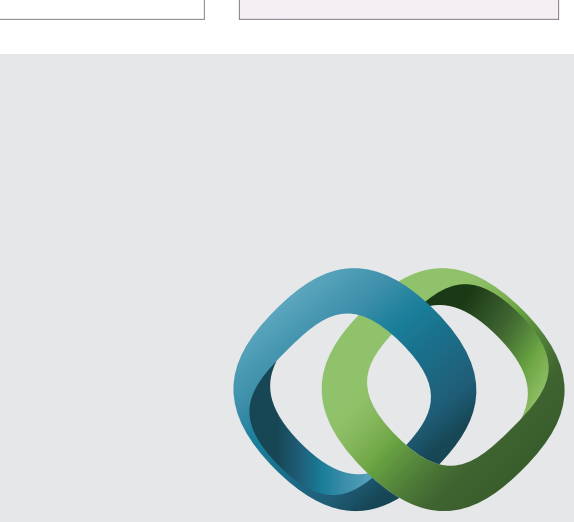

\section{Hindawi}

Submit your manuscripts at

http://www.hindawi.com
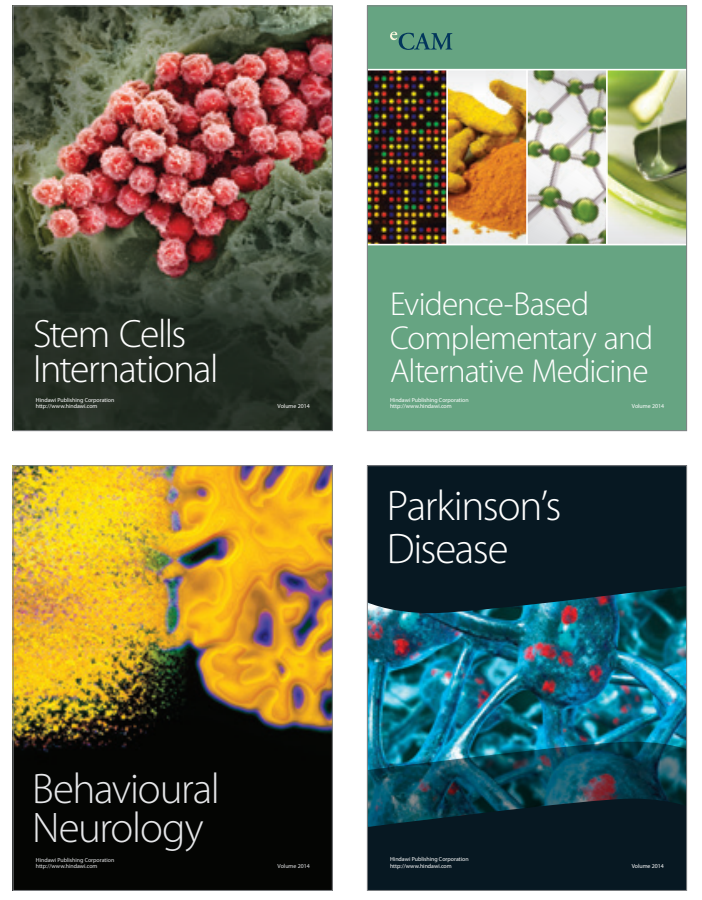
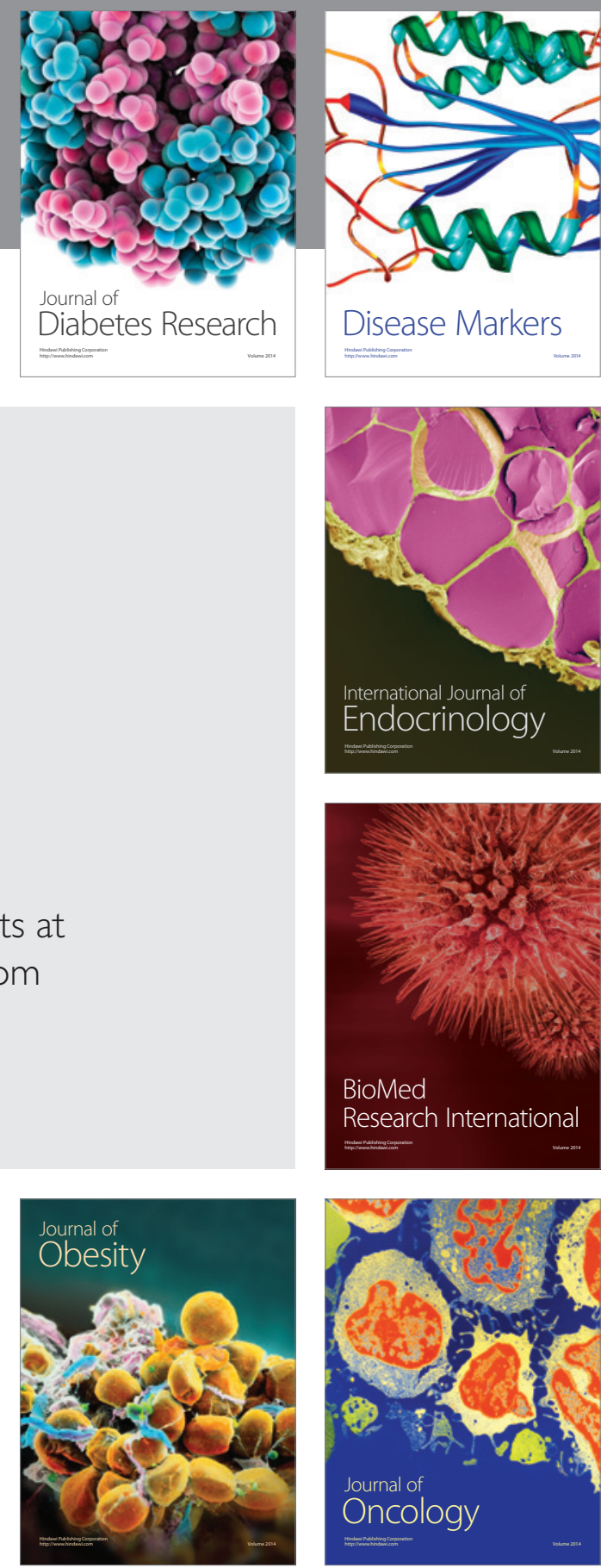

Disease Markers
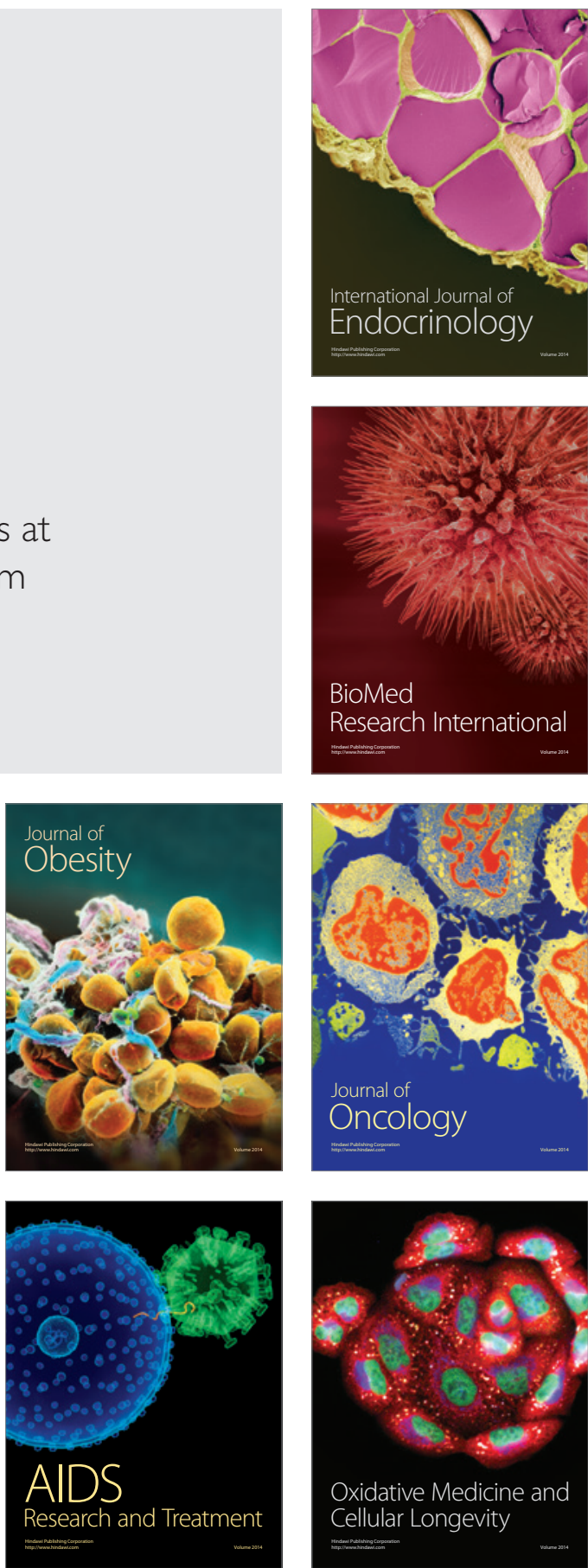